\title{
Introduction
}

The following paper discusses the use of Information and Communication Technologies (ICTs) by mental health nurses as part of their clinical practice. It seeks to illustrate the benefits to be had from such technologies and why it is important that mental health nurses should engage with them as part of clinical practice. Understanding how ICTs currently contribute to practice and generate clinical value should be considered the step first in this process before looking at the wider implications and use of such technologies in promoting service innovation and improvement (the why mental health nurses should get involved?). Whilst the reported benefits of ICTs are well-documented, improved information management, access to health services, quality improvement and cost containment, their effects, it is argued here, have yet to be fully realised by mental health nurses as part of their day-to-day practice. How much of this can be attributed to the ICTs themselves or what seems to be a reluctance by clinicians to engage with such technologies remains unclear and open to debate.

\section{Information and Communication Technologies}

ICTs embody 'all digital technologies that support the electronic capture, storage, processing and exchange of information' (Rouleau et al. 2015) in support of clinical practice. They have been around since the second half of the 1980s and have been used in a variety of forms. ICTs are used to store care plans, chart and record clinical and patient activity, as well as provide an educational and evidence based resource. They allow for the access, processing and interpretation of patient data and through this have the potential to transform clinical environments (Tello \& Barbazza 2015). ICTs shape practice by the way in which care is organized and delivered and by the way in which clinicians communicate with each other. They are powerful and pervasive and have a direct impact on patient experience through the enablement of entirely new models of care and ways of working (Car et al. 2008). ICTs that contain alerts on a client's mental health status and risk and facilitate communication between clinicians and providers have proved to enhance care and patient safety, especially at the inpatient and community interface. 
The potential use of ICTs continues to grow with the development of high speed and wireless connections, decreasing cost and the technology boom in personalised digital assistants (PDAs), making such devices smaller, and more portable (While \& Dewsbury 2011). This technological boom runs alongside advances in 'smart technology' whereby different technologies and devices can 'talk to each other', allowing for the creation of care environments that not only monitor the safety and wellbeing of the client but respond to their changing needs in 'real time', for example, creating safe environments for people living with dementia (Bowes et al. 2013). Despite these advices, there is evidence to suggest that such technologies remain underutilised and disliked in mental health practice (Johansson et al. 2014). There would seem to be a number of reasons for this, none more so than the way that ICTs were first introduced and promoted in clinical practice.

There is a phenomenon called the Solow computer paradox (also known as the productivity paradox) that suggests that, as more investment is made in information and communications technology, worker productivity tends to go down rather than up. This observation, supported by empirical evidence, is counter intuitive and worthy of investigation. The concept itself was first described in 1987 by the economist and author Robert Solow, who went on to win the Nobel Prize for his work, who stated, 'You can see the computer age everywhere but in the productivity statistics' (Solow 1987). Numerous hypotheses have been put forward as to why this should be. Perhaps the most prominent of these is that by Erik Brynjolfsson and Andrew McAfee (2014), who suggest that technological advances increase productivity only after a long lag; that there needs to be a bedding in period before the true benefits of such technologies are accessed and realised. But how long is the wait? The hugely ambitious NHS National Programme for Information Technology (NPfIT), subsequently renamed Connecting for Health, has been and gone. Introduced in 2002 the programme was the largest public sector IT projects ever attempted in the UK with an original budget of six billion pounds. After a history marked by delays, stakeholder opposition and implementation issues, the programme was dismantled in 2011 having nearly doubled in cost (Campion-Awwad et al. 2014). 
For many nurses the initial attraction of ICTs was the misguided perception that such technology would be a solver of problems; after all that was one of the things that it was promoted on. It was envisaged that the introduction of ICTs would provide clinicians with the information that they needed to do their job and to ensure that patients, carers and the public had the information necessary to make decisions about their care and treatment, and to influence the shape of health services generally (Hendy et al. 2005). These nurses thought that ICTs would demonstrate how hard they worked, would simplify care planning and save time spent on managerial activity, such as drawing up the duty rota, giving them more time to spend with their patients. However, none of these assumptions bear scrutiny.

Despite advances in the design, function and availability of ICTs and computerised systems, they often fall short of their projected contributions when implemented in the health care setting and are unable to provide the type of information required of them by the mental health nurse in clinical practice. The rapidly changing context in which health care is provided and delivered continues to challenge the most experienced of clinicians. Modern technology does not always keep up with these changes or their introduction might be ill timed and not correspond to the needs of the day. The benefits of ICTs are not always apparent, as staff struggle to 'get to grips' with the new technology and clinician frustration can overshadow any progress made. Such systems are viewed with 'suspicion' and counterintuitive to the humanistic qualities required of the mental health nurse in practice. Too much time is 'spent in the office working on the computer, rather than with the service user'. Email was to transform the way in which clinicians and services communicated with each other, however this just seems to have added to the stress experienced by nurses in trying to keep up with the sheer volume of intranet 'traffic'. It was always envisaged that using electronic communication would support new forms of collaboration between healthcare professionals, and enhance care by making information available at a time that it was required in a format that could be used to support clinical practice.

Part of the despondency felt by nurses towards ICTs is the perception that the information they gather seldom relates to their practice. For example, where activity 
data for a ward is collected implications for practice are either not identified or the nurses do not control the staffing budgets to respond to the data generated and make changes as required. The ability of ICTs to radically enhance patient care delivery is not questioned here, however their uptake and use largely remains limited to the storage and retrieval of organizational data and information. These systems give little priority to nurse specific information and are more concerned with the collection of formal organizational data for audit purposes, and are often replicas of the original paper process in a 'mechanised' form. One of the fundamental changes when conventional paper-based records are replaced with electronic data records 'involves the ability to process the data in that record for different purposes' (Van Der Lei, 2002). Ideally, nurses should be able to engage with technologies as a process of their work and develop their own interpretation of the information provided by ICTs in order to construct their own meaning of that information for use in clinical practice. Instead, we remain with ICTs that do not always 'talk' to each other (Meinert, 2004), that are slow to process information and prone to breaking down (Ajami \& BagheriTadi, 2013), adding further to the frustration of nurses.

Mental health nursing stresses the interpersonal relationship between people and is concerned with human growth and emotional wellbeing (Barker 2008). It emphasises the diversity and uniqueness of service user experiences with the context of a matrix of bio-psycho-social considerations impacting upon the immeasurable day-to-day life quality of those for whom nurses care for. ICTs, at first, were programmed to emulate or resemble existing work based processes aimed at 'speeding up' operational support. This, more often than not, equated to the existing paper based version of a document put into an electronic format. Increasingly, computer systems are being designed towards minimizing the entry of information by reducing the amount of data that health care professionals record electronically and increasingly favour a 'tick-box' approach in order to deliver formal organizational information requirement. (Stevenson et al, 2010). This assumes that nurses' information requirements are captured by ICTs, that nursing practice stands still and that nurses have control over the use of information in the system. Meeting the formal demands for information from the organization is important to any health care professional, likewise meeting 
the professional demands for clinical information for nursing is important to the profession itself. Failing to capture nursing information through any form of clinical communication is detrimental to professional obligation and practice. If tightly structured technology is used, to manage and process information, then the richness of nursing will be lost to the profession, much to its detriment (Stevenson et al, 2010). It is important that the wealth of data expounded in nursing not be lost in the reduction of data codes with which the computer can deal with more easily. Detailed customization is needed for any service. This is not in technical detail about planning or software but rather in defining the operational tasks and educational support that ICTs should undertake, and allow the implementation of systems that will actually match what it is they do, to what is required of them by those in clinical practice (De Veer et al, 2011).

User acceptance has been identified as the pivotal factor in explaining the success or failure of ICTs in clinical practice (Koivunen 2009). Where computer systems have been developed with the nurse in mind they have tended to incorporate an element of decision-support. Clinical decision-support has been defined as: '... active knowledge systems that use two or more items of patient data to generate carespecific advice' (Van Bemmel \& Musen 1997). The authors have highlighted the word advice in italics to stress the point that such systems should support not replace clinician decision-making. The development of computerized decision-support systems has stressed the need for standardization as a means of providing baseline information to evaluate practice processes and patient outcomes and often model medical knowledge. The prescriptive approach to decision making through the use of standardized practice protocols restricts nurses' ability to creatively explore alternative approaches to clinical practice problems. For example, ICTs are typically developed from standadised clinical protocols. These systems can only advise nurses based on the knowledge that they contain. Nurses who come to rely exclusively on the advice of such systems may not be able to discriminate when the computer support system advice may not be applicable and they should seek other knowledge resources. Clinical practice standardization also underscores an assumption that clinical practice variation represents resistance to change when it may actually reflect 
legitimate variation based on the nurses' use of professional artistry in new and uncertain clinical situations. Whilst it might be appropriate for other health care professionals to record their intermittent care episodes within a minimalist electronic framework, it is considerably more difficult for nurses if they are to retain their holistic, qualitative frameworks to support the rich information they receive from patients and relatives. Clearly, while many aspects of ICTs can help and inform the practice of nurses, standardized clinical practice is not one that reflects the requirements of either nurses or service users.

\section{Time for a rethink}

ICTs are not going to go away and maybe it is now time for a rethink as to their use. Accessing database information is not only important but also essential. Healthcare is dependent on information for decision-making and it is important that nurses are 'capable of practicing in an environment where technology continues to increase in amount and sophistication' (American National League for Nursing 2008). ICTs are to be found in all elements of modern life and it is now hard to image a world without them. A key feature that distinguishes our relationship with ICTs from that of the past is that we are living in a technological age were their use is commonplace. Increasingly, patients are presenting clinicians with things they have found online regarding their illness or condition; requesting medication that is being championed in one country and not another; accessing self-help apps, or indeed, therapies online, there is growing evidence as to the effectiveness of computer-aided cognitive - behavioral therapy for example, and finally, psycho-educational and psychosocial support (Hardey, 1999, Eysenbach, 2001). What is needed is a rethink as to how mental health nurses can incorporate ICTs more readily into their practice. Mental health nursing is no longer aimed primarily at caring for the mentally ill, but also promoting mental health by helping the patient to utilize his resources and potential to meet their physical, social and psychological needs, so that they may return to the community from which they came better equipped to cope with the demands of daily life (Cattan and Tilford, 2006). Therefore, the use of ICTs as part of clinical practice should support these goals. The challenge is to provide nurses and service users with ICTs that support the humanistic aspects of clinical practice in a fashion that allows flexibility in their 
use, dependent on the needs of the service user at a given point in time. The nurse's role then becomes one of creating opportunities for the use of ICTs in support of clinical practice.

Advancements in smart technology have opened new opportunities in mental health care. There are now a number of downloadable mental health software applications (apps) for use with mobile phones and other portable devices covering a wide range of treatment interventions and assessment tools (Cotton et al. 2014). Complete courses of cognitive behavioral therapies are available for use by the service user for example. Mobile phone applications such as Skype have opened new avenues and possibilities in telemental health, whereby engagement with the service user is done over distance, cutting down on the travelling times and cost, means that the mental health nurse can 'see' more patients in a day. There are apps to aid diagnosis and assessment, to monitor treatment progress, and evaluate the effectiveness of interventions. There are life style apps giving advice on healthy living, and apps to help people quit smoking and lose weight. Perhaps the biggest strength of such devices and technology is the breaking down of social isolation. Australia has had a large degree of success of combating social isolation in the elderly population with the issuing of Ipads to those of pensionable age and living away from their relatives (Feist et al. 2012).

There already exists a vast amount of literature about change methods and strategies, which transverse the boundaries of many academic disciplines and management practice. An awareness of the advantages to achieve a specific development is essential to any success and sustaining significant progress (Gagnon et al, 2012). Whatever intervention is pursued, it will inevitably be influenced by those that are providing it. In order to achieve practice improvement there needs to have been a positive change which measurably improves the efficiency or effectiveness of the service. A problem has been identified and a recognizable improvement attained. It has, what we have termed, 'contestability' that any improvement can be both measured and articulated, not just by those involved in carrying out the change, but also those receiving the service. Acceptance of any system requires a change of thinking and incorporation of meaning. In other words, the technology is connected 
to who they are, what they care about, and how they perceive and know. Mental health nurses need to get involved and have an understanding as to what is available to service users and how service users are using ICTs as part of their daily living. This is not in technical detail about planning or software application but defining the operational and practice support that ICTs should undertake, and promoting and championing such technologies that have proven service application, and that actually match what it is they do, to what is required of them by those in clinical practice, and most importantly, that support patient wellbeing and care. (While and Dewsbury, 2011). How we manage our information determines what we can do and how well we do it. Properly programmed, computers are capable of dramatically enhancing nursing practice. In order to achieve this, nursing, as a profession needs to be allowed to not only be involved in, but also actively manage its information and technological requirements (De Veer et al, 2011). When reviewing the wider literature, the use of ICTs as an intervention is singularly absent. The flexibility that such technology offers is fundamental to its success, it's just that we have to capitalize on this. Nurses could well have to rethink their role in line with technological advances for instance. There is the potential for improved communication through the sharing of information, partnership working, the creation of online service user groups and e-health interventions designed around the service user for use at a time convenient to them (Eysenbach, 2001).

\section{Conclusion}

There is no doubt that information technology has moved on since their inspection and that we are living in a technological advanced age. If such technological systems are to meet the needs of the nurse in clinical practice, then the nurse needs to rethink how they use ICTs in meeting service user demands. The nurse's role becomes one of creating opportunities for the use of ICTs in support of clinical practice. Nurses should be skilled enough to manage their information requirements in a way that sustains their specific area of practice for the betterment of their patients. Information technologies should enhance the delivery of patient care, not detract from it. 


\section{References}

Ajami, S. and Bagheri-Tadi, T. (2013). Barriers for Adopting Electronic Health Records (EHRs) by Physicians. Acta Inform Med. 2013; 21(2) p129-134.

American Nurses Association (2008). Nursing informatics: Scope and standards of practice. Washington DC: The Author.

Barker, P. (Ed) (2008). Psychiatric and Mental Health Nursing: The craft of caring. $\left(2^{\text {nd }}\right.$ Ed.) London: CRC Press

Bowes A., Dawson, A. and Greasley-Adams, C. (2013). Literature review: the cost effectiveness of assistive technology in supporting people with dementia. Report to the Dementia Services Development Trust. University of Stirling.

Brynjolfsson, E. and McAfee, A. (2014). The Second Machine Age: Work, Progress, and Prosperity in a Time of Brilliant Technologies. W. W. Norton \& Company: London.

Car, J., Black, A., Anandan, C., Cresswell, K., Pagliari, C., McKinstry, B., Procter, R., Majeed, A. and Sheikh, A. (2008). The Impact of eHealth on the Quality \& Safety of Healthcare: A Systemic Overview \& Synthesis of the Literature. Report for the NHS Connecting for Health Evaluation Programme March 2008. Imperial College London.

Campion-Awwad, O., Hayton, A., Smith, L. and Vuaran, M. (2014). The National Programme for IT in the NHS A Case History. MPhil Public Policy 2014, University of Cambridge.

Cattan, M. and Tilford, S. (2006). Mental Health Promotion: A Lifespan Approach. 
McGraw Hill: Open University Press.

Cotton, R., Irwin, J., Wilkins, A. and Young, C. (2014). The futures digital: Mental health and technology. London: Mental Health Network: NHS Confederation.

De Veer, A.J., Fleuren, M.A.H., Bekkema, N., and Francke, A.L. (2011). Successful implementation of new technologies in nursing care: a questionnaire survey of nurseusers. BMC Medical Informatics and Decision Making. 11:67.

Eysenbach, G. (2001). What is e-health? Journal of Medical Internet Research. 3(2): e20.

Feist, H. R., Parker, K. and Graeme, H. (2012). Older and Online: Enhancing social connections in Australia rural places. The Journal of Community Informatics. 8, 1, Feb 2012. Available at: <http://ci-journal.net/index.php/ciej/article/view/818>. Date accessed: 16 Apr. 2017.

Gagnon, M.P., Desmartis, M., Labrecque, M., Car, J., Pagilari, C., Pluye, P., Fremont, P., Gagnon, J., Tremblay, N. and Legare, F. (2012). Systematic Review of Factors Influencing the Adoption of Information and Communication Technologies by Healthcare Professionals. Journal of Medical Systems. Feb; 36(1) p241-277.

Hardey, M. (1999). Doctor in the house: the internet as a source of lay health knowledge and the challenge to expertise. Sociology of Health and IIIness. 21 (6) p820835.

Hendy, J., Reeves, B.C., Fulop, N., Hutchings, A. and Masseria, C. (2005). Challenges to implementing the national programme for information technology (NPfIT): a 
qualitative study. BMJ, 6, 331 (7512): $331-336$.

Johansson, P., Petersson, G., Saveman, B-I., and Nilsson, G. (2014). Using advanced mobile devices in nursing practice - the views of nurses and nursing students. Health Informatics Journal, 20, 3, 220 - 231.

Koivunen, M. (2009). Acceptance and use of information technology among nurses in psychiatric hospitals. Painosalama Oy - Turku, Finland 2009 ISBN 978-951-29-3818-6 (PDF).

Meinert DB. Resistance to Electronic Medical Records(EMRs): A Barrier to Improved Quality of Care. Informing Science: International Journal of an Emerging Transdiscipline. 2004; 2: 493-504.

Rouleau, G., Gagnon, M-P. and Cote, J. (2015). Impacts of information and communication technologies on nursing care: an overview of systematic reviews (protocol). BioMed Central, Systematic Reviews, 4:74. Published online 2015 May 23. 10.1186/s13643-015-0062-y Last Accessed 02.04.2017

Solow, R. M. (1987). "We'd Better Watch Out" review of Manufacturing Matters: The Myth of the Post-Industrial Economy, by Stephen S. Cohen and John Zysman, New York Times, July 12, 1987.

Stevenson, J.E., Nilsson, G.C., Petersson, G.I., Johansson, P.E. (2011). Nurses' experience of using electronic patient records in everyday practice in acute/inpatient ward settings: a literature review. Health Informatics Journal. 16(1) p63-72. 
Tello, J. and Barbazza, E. (2015). Health services delivery: a concept note. WHO Europe: Geneva.

Van der Lei, J. (2002). Closing the Loop between Clinical Practice, Research, and Education: The Potential of Electronic Patient Records. Methods Inf Med, 41: 51 - 54.

While, A. and Dewsbury, G. (2011). Nursing and information and communication technology (ICT): a discussion of trends and future directions. International Journal of Nursing Studies, 48:1302-10. 